\title{
Systems pharmacology-based integration of human and mouse data for drug repurposing to treat thoracic aneurysms
}

\author{
Jens Hansen, ${ }^{1}$ Josephine Galatioto, ${ }^{1}$ Cristina I. Caescu, ${ }^{1}$ Pauline Arnaud, ${ }^{2,3}$ Rhodora C. Calizo, ${ }^{1}$ \\ Bart Spronck,, ${ }^{4}$ Sae-II Murtada, ${ }^{4}$ Roshan Borkar, ${ }^{5}$ Alan Weinberg, ${ }^{6}$ Evren U. Azeloglu, ${ }^{1}$ \\ Maria Bintanel-Morcillo, James M. Gallo, ${ }^{5}$ Jay D. Humphrey, ${ }^{4}$ Guillaume Jondeau, ${ }^{2,3}$ \\ Catherine Boileau, ${ }^{2,3}$ Francesco Ramirez, ${ }^{1}$ and Ravi lyengar ${ }^{1}$ \\ 'Department of Pharmacological Sciences and Institute for Systems Biomedicine, Icahn School of Medicine at Mount \\ Sinai, New York, New York, USA. ²Département de Génétique et Centre de Référence Maladies Rares Syndrome de \\ Marfan et Pathologies Apparentées, Assistance Publique-Hôpitaux de Paris, Hôpital Bichat, Paris, France. ${ }^{3}$ LVTS, \\ INSERM U1148, Université Paris Diderot, Hôpital Bichat, Paris, France. ${ }^{4}$ Department of Biomedical Engineering, Yale \\ University, New Haven, Connecticut, USA. ${ }^{5}$ Department of Pharmaceutical Sciences, State University of New York at \\ Buffalo, Buffalo, New York, USA. ${ }^{6}$ Department of Population Health Science and Policy, Icahn School of Medicine at \\ Mount Sinai, New York, New York, USA.
}

\begin{abstract}
Marfan syndrome (MFS) is associated with mutations in fibrillin-1 that predispose afflicted individuals to progressive thoracic aortic aneurysm (TAA) leading to dissection and rupture of the vessel wall. Here we combined computational and experimental approaches to identify and test FDA-approved drugs that may slow or even halt aneurysm progression. Computational analyses of transcriptomic data derived from the aortas of MFS patients and MFS mice (Fbn 1 mgR/ ${ }^{m g R}$ mice) predicted that subcellular pathways associated with reduced muscle contractility are key TAA determinants that could be targeted with the GABA ${ }_{B}$ receptor agonist baclofen. Systemic administration of baclofen to $F b n 1^{m g R / m g R}$ mice validated our computational prediction by mitigating arterial disease progression at the cellular and physiological levels. Interestingly, baclofen improved muscle contraction-related subcellular pathways by upregulating a different set of genes than those downregulated in the aorta of vehicle-treated Fbn $1^{\text {mgR/mgR }}$ mice. Distinct transcriptomic profiles were also associated with drug-treated MFS and wild-type mice. Thus, systems pharmacology approaches that compare patient- and mouse-derived transcriptomic data for subcellular pathway-based drug repurposing represent an effective strategy to identify potential new treatments of human diseases.
\end{abstract}

Conflict of interest: The authors have declared that no conflict of interest exists.

Author contributions: JH, JG, and CIC contributed equally to the study. FR and RI are co-senior authors.

Copyright: @ 2019 American Society for Clinical Investigation

Submitted: January 23, 2019

Accepted: April 25, 2019

Published: June 6, 2019.

Reference information: /CI Insight. 2019;4(11):e127652. https://doi. org/10.1172/jci.insight.127652

\section{Introduction}

The therapeutic promises of precision medicine largely rely on our ability to distinguish molecular determinants of pathogenesis from correlative, compensatory, or other concurrent physiological processes (1). Diseases like cancer that arise from multiple genomic alterations can often be defined by individual determinants that enable targeted therapies (2). However, the situation is more complicated for other progressive diseases. Many molecular determinants can control disease progression even in monogenic disorders, thus requiring systems-level approaches to identify underlying mechanisms. Systems pharmacology views disease as a malfunction of integrated molecular interactions - i.e., networks of multiple subcellular pathways (SCPs) — and drug treatment as a method to restore physiological response of disease-centered networks by targeting one or more of their components (3). Analyses of transcriptomic data for underlying SCPs provides an unbiased strategy to identify biological targets in disease-related regulatory networks, and then use this information to predict known drugs and compounds that can be repurposed for new therapeutic applications.

Thoracic aortic aneurysms (TAAs) are life-threatening pathologies characterized by progressive vessel dilation associated with smooth muscle cell (SMC) dysfunction, occasional localized inflammatory infiltrates, and severe maladaptive extracellular matrix (ECM) remodeling that, together, predispose the arterial wall to dissection and rupture leading to premature death (4). Consistent with the progressively degenerative nature 
of the disease, inherited forms of TAA are accounted for by mutations in molecules involved in supporting tissue integrity and homeostasis, such as components of the ECM, SMC contractile apparatus and its mediators, and TGF- $\beta$ signaling pathways (4). Yet, the molecular mechanisms and SCPs that translate these different genetic defects into a seemingly identical pathology are not fully understood. As a result, prevention of untimely death from TAA complications currently relies on early detection by imaging and prophylactic repair by surgery. Unfortunately, disease progression is highly heterogeneous, clinical outcome is unpredictable, therapeutic options are limited, and surgical intervention carries significant morbidity/mortality risks.

TAA with ensuing dissection and rupture of the vessel wall is the clinical hallmark of Marfan syndrome (MFS), a relatively common connective tissue disease associated with mutations in the gene that codes for the multifunctional ECM glycoprotein fibrillin-1 (4, 5). Fibrillin-1 assemblies (microfibrils and elastic fibers) impart specific physical properties to tissues, distribute mechanical forces within and across them, communicate to multiple types of vessel wall cells through integrin receptors, and modulate local bioavailability of ECM-bound latent TGF- $\beta$ complexes (5). In spite of significant research effort, the molecular pathogenesis of arterial disease in MFS remains unresolved, therefore hindering advances in drug therapy. Earlier studies of MFS mice with nondissecting TAA ( $F b n 1^{C 1039 G /+}$ mice) have correlated aneurysm onset and progression with increased TGF- $\beta$ signaling in the media stimulated by improper angiotensin II (AngII) type I receptor (AT1r) activity $(6,7)$. More recent findings indicate a more complex disease mechanism involving the gradual stratification of stress-stimulated interactions among different cell types and multiple regulatory pathways, of which the AT1r and TGF- $\beta$ signaling pathways are a critical subset (8-14).

An overview of regulatory pathways and networks associated with a given pathology can often be obtained by examining changes in gene expression during disease progression. Systems pharmacology approaches that consider drug targets as nodes within cellular regulatory networks can use differentially expressed genes (DEGs) to predict dysregulated SCPs that underlie cell-level mechanisms (1, 3). Further, computational analyses of the pharmacologically induced perturbations of gene expression listed in the Connectivity Map (CMap) database can predict drugs to be repurposed to normalize dysregulated SCPs (15). By revealing that SCPs associated with muscle cell contractility are downregulated in both aortic SMCs isolated from MFS patients and aortic tissue harvested from MFS mice, this strategy enabled us to predict that the $\mathrm{GABA}_{\mathrm{B}}$ receptor agonist baclofen could restore normal activity of these TAA-related SCPs. To test our computational prediction, we performed a series of in vivo and ex vivo analyses that demonstrated a statistically significant mitigation of TAA pathology at the cellular and physiological levels in baclofen- versus vehicle-treated MFS mice. Hence, we conclude that alterations in muscle contractility processes contributing to TAA development in MFS can be therapeutically targeted through $\mathrm{GABA}_{\mathrm{B}}$ receptors.

\section{Results}

Our study was organized into 3 successive lines of investigation. First, we identified shared SCPs from transcriptomic analyses of SMCs isolated from the dilated aorta of MFS patients and aortic tissue harvested from Fbn$1^{m g R / m g R}$ mice, a validated animal model of early-onset progressively severe MFS (16). Second, we used the gene expression profiles listed in the CMap database (15) to predict FDA-approved drugs that could normalize the dysregulated SCPs in common between human and mouse aortic samples. Third, we tested a top-ranked drug prediction for its ability to modify TAA pathology in $F b n 1^{m g R / m g R}$ mice and determined if the drug acted through the subcellular mechanisms predicted to be shared by the diseased aortas of MFS patients and MFS mice.

Impaired SMC contractility is associated with TAA in both MFS patients and mice. RNA sequencing (RNASeq) was used to identify genes differentially expressed in the aortas of $F b n 1^{m g R / m g R}$ (MFS) mice versus wild-type (WT) littermates, and in aortic SMCs of MFS versus non-MFS patients (Figure 1, A and B, and Supplemental Tables 1 and 2; supplemental material available online with this article; https://doi. org/10.1172/jci.insight.127652DS1). MFS patients and mice were comparable with respect to the phenotypic severity as opposed to the disease stage in which the tissues had been harvested. Gene Ontology (GO) SCP enrichment analysis of upregulated and downregulated genes revealed that SCPs related to actin cytoskeleton dynamics and muscle contractility were top-ranked in the downregulated genes of both human SMCs and mouse aortas (Figure 1, C and D, and Supplemental Tables 3 and 4). By contrast, a similar analysis found no top-ranked disease-relevant SCPs in common between the upregulated genes of human and mouse aortic specimens (Supplemental Figure 1, A and B). Using the top-ranked downregulated SCPs associated with muscle contraction as seed nodes in the GO topology, we identified all related SCP ancestors and offspring that contained at least 20 genes and were also related to SMC contractility 


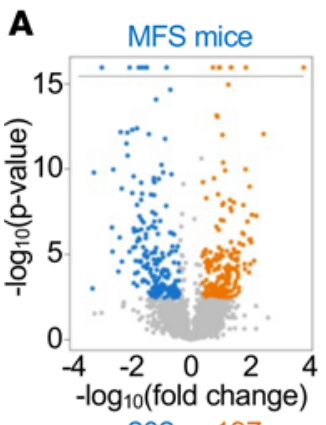

202197

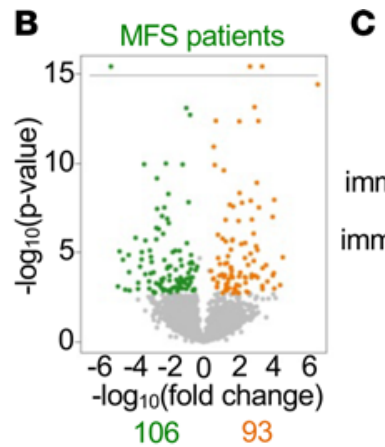

10693
C

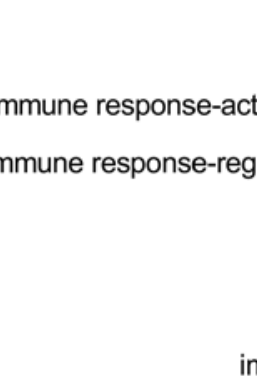

\section{Downregulated SCPs in MFS mice}

$T$ cell receptor signaling pathway antigen receptor-mediated signaling pathway regulation of muscle system process regulation of muscle contraction face receptor signaling pathway regulation of system process
gulating cell surface receptor signaling pathway positive regulation of interleukin-2 production positive regulation of $T$ cell activation regulation of $\mathrm{T}$ cell activation actin-myosin filament sliding muscle filament sliding nephron tubule development immune response-activating signal transduction renal tubule development

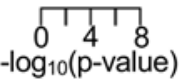

D

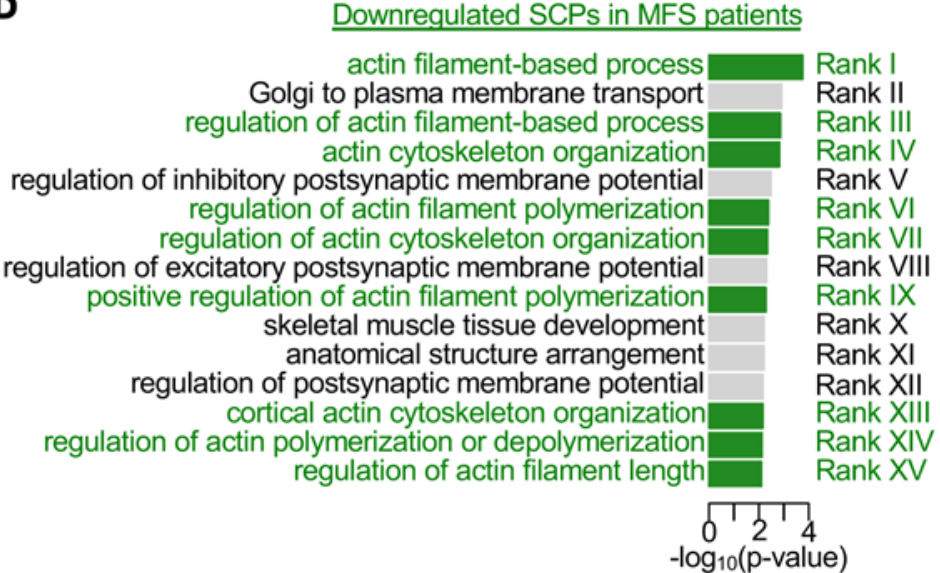

E

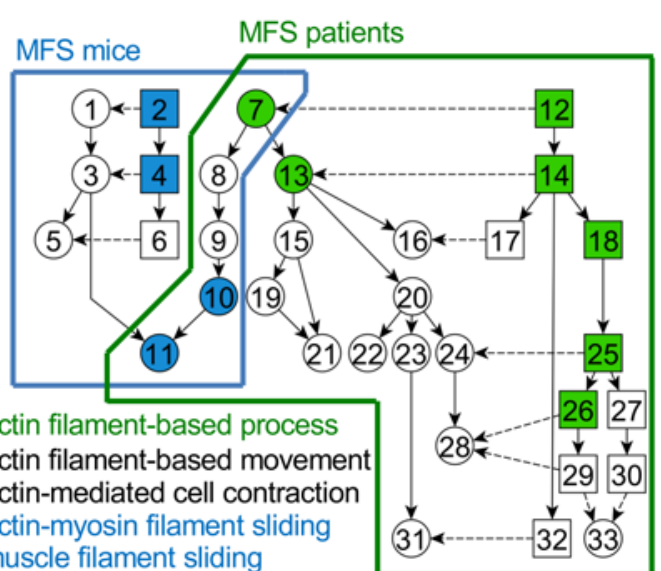

Figure 1. DEG analyses correlate defective muscle contractility with TAA progression in MFS mice and patients. Volcano plots of DEGs in (A) aortas of MFS mice sacrificed at P16 ( $n=3$ mutant mice and $n=3$ WT mice) and (B) aortic SMCs of MFS patients aged between 22 and 32 years ( $n=3$ MFS patients and $n=3$ non-MFS organ transplant donors). The $\log _{2}$ of fold change is plotted against the negative $\log _{10}$ of the $P$ value; blue/green dots indicate significantly downregulated genes, and orange dots indicate significantly upregulated genes. Dots above the horizontal gray line indicate genes whose minus $\log _{10}(P$ value) was calculated as infinity (since its $P$ value was below the lowest number that is supported by Cuffdiff and therefore given as zero). Blue/orange or green/orange numbers give total counts of significantly down- or upregulated genes in mouse and human samples, respectively. Top-ranked (according to $P$ values) SCPs related to muscle contractility (blue or green boxes) from GO enrichment analysis of mouse aorta (C) or human SMC (D) downregulated genes. Integration of the enrichment results into GO-SCP topology identifies 2 muscle contractility-related subnetworks (E) that have 5 SCPs in common. SPCs predicted to be downregulated in MFS aortas and human aortic SMCs are in blue and green, respectively, and intermediate SCPs are in white. Solid lines connect parents with their children processes ("is_a" or "part_of" GO relationship), dashed lines indicate regulatory relationships ("regulates" GO relationship). Regulatory SCPs are visualized as rectangles, all other SCPs as circles. Identity of network components can be found in Supplemental Table 5.

(Figure 1E and Supplemental Table 5). Importantly, network analysis revealed that the mouse (blue) and the human (green) downregulated subnetworks shared 5 (seed or intermediate) GO-SCPs related to muscle contractility (Figure 1E). As our study was being completed, Lino Cardenas at al. (17) reported that genes coding for contractile proteins are similarly repressed in the aorta of MFS mice with nondissecting TAA.

Computational identification of baclofen as a potential anti-TAA drug. Next, we screened the CMap gene expression database (15) to predict FDA-approved drugs that could upregulate the 5 muscle contractility-related SCPs found in common between the downregulated subnetworks of the mouse and human aortic specimens (Figure 1E). Each of the CMap drugs had originally been tested under several different conditions (i.e., cancer cell lines, drug concentration, and treatment length) listed in the database as separate experiments. We subjected the upregulated human genes in the CMap database to GO-SCP enrichment analysis under the assumption that SCPs most significantly enriched in upregulated genes best reflect the main action of a given drug. It follows that a drug was not considered further if the associated top-ranked SCP was not disease relevant, even if a high-significance disease-relevant SCP was low-ranked. This approach identified 12 candidate drugs for which upregulation of a disease-relevant SCP was predicted at the top rank (Figure 2A and Supplemental Table 6); of them, only 4 were consid- 
A

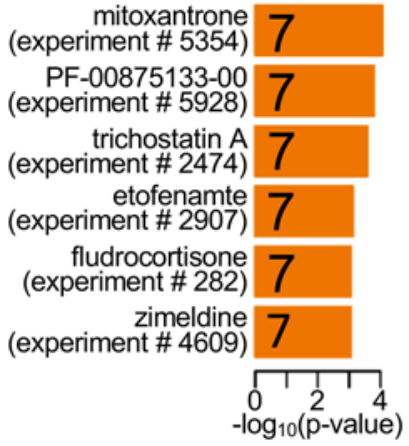

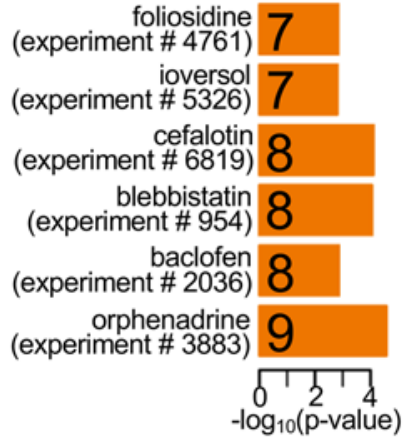

B

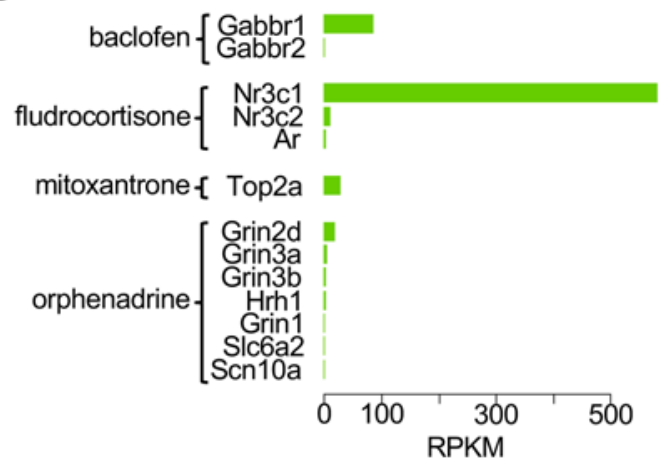

C

\section{Upregulated SCPs - baclofen (experiment \# 2036)}

actin filament-based movement positive regulation of growth hormone secretion regulation of defense response to virus

regulation of acute inflammatory response to antigenic stimulus negative regulation of inflammatory response to antigenic stimulus regulation of mesoderm development negative regulation of acute inflammatory response regulation of growth hormone secretion actin-myosin filament sliding muscle filament sliding female pregnancy negative T cell selection negative thymic $T$ cell selection actin-mediated cell contraction sensory perception of pain

\section{Rank I}

Rank II

Rank III

Rank IV

Rank V

Rank VI

Rank VII

Rank VIII

Rank IX

Rank X

Rank XI

Rank XII

Rank XIII

Rank XV

D

Upregulated SCPS - baclofen (all experiments combined)

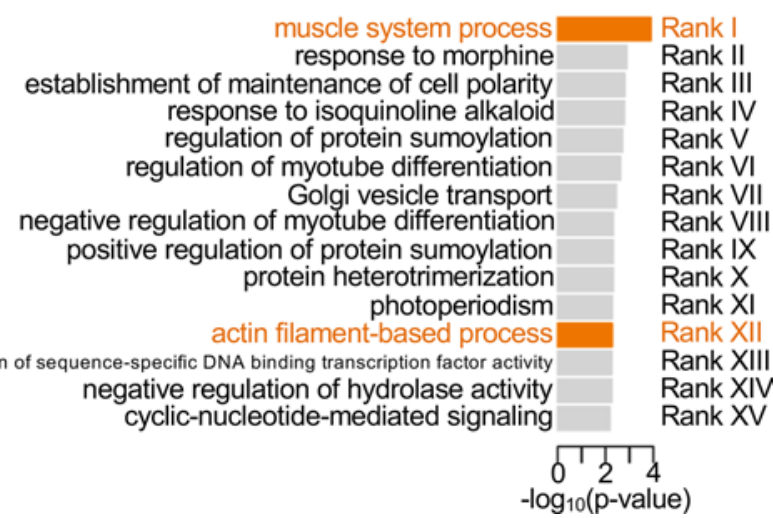

Figure 2. Baclofen is a predicted potential treatment for TAA. (A) Top-ranked potential drug candidates identified from GO-SCP enrichment analysis of upregulated genes in the CMap database. Bars indicate the minus $\log _{10}(P$ values) of the identified treatments, numbers correspond to the SCPs in the subnetworks of Figure 1E. (B) Target proteins of the indicated drugs annotated in the Drug Bank database (see also Supplemental Table 7); green bar graphs on the right indicate the expression levels of the corresponding mouse genes in the P16 MFS aorta. (C) CO-SCPs identified in the top-ranked list of SCPs derived from baclofen-stimulated genes in the annotated drug treatment baclofen number 2036 of the CMap database (see Supplemental Figure 2A and B). (D) Top-ranked muscle contractility-related CO-SCPs in the combined list of upregulated genes in all baclofen treatments of the CMap database. In panels $\mathbf{C}$ and $\mathbf{D}$, muscle contractility-related SCPs are in orange.

ered further because they had an annotated protein target in the database (Figure 2B and Supplemental Table 7). Based on the relatively high expression of their respective targets in the aorta of MFS mice, this shorter list of top-ranked drugs was narrowed down to the steroid fludrocortisone and the muscle relaxant baclofen (Figure 2B). However, fludrocortisone was excluded from further analyses because of its potential to induce iatrogenic arterial hypertension, the major risk factor for thoracic aortic disease (14).

Baclofen is a highly selective drug for $\mathrm{GABA}_{\mathrm{B}}$ receptors, as evidenced by 17 supporting references in the Drug Bank database (http://www.drugbank.ca). Two additional computational analyses further strengthened the predicted stimulatory effect of $\mathrm{GABA}_{\mathrm{B}}$ receptor agonism on muscle contractility. In the first approach, manual inspection of the top 15 upregulated GO-SCPs listed in the CMap database as experiment 2036 identified 4 other muscle cell-related mechanisms (Figure 2C and Supplemental Table 8). By contrast, no muscle function-related SCPs were predicted through a similar analysis of the top 15 downregulated GO-SCPs (Supplemental Figure 2A and Supplemental Table 8). In the second approach, GO-SCP enrichment analysis of the combined list of upregulated genes in the 5 different baclofen experiments of the CMap database identified 2 top-ranked muscle cell-related SCPs (Figure 2D and Supplemental Table 9). Parallel inspection of the combined list of downregulated genes identified "Actin filament de-polymerization" as the top SCP, consistent with the destabilizing effect of this mechanism on muscle function (Supplemental Figure 2B and Supplemental Table 9). Collectively, our analyses supported the prediction that $\mathrm{GABA}_{\mathrm{B}}$ agonism may represent a potential treatment option against TAA progression in MFS.

In vivo validation of baclofen efficacy in slowing TAA progression in MFS mice. To test the above prediction, we compared TAA progression in MFS mice treated with either baclofen or vehicle. Baclofen was system- 
A
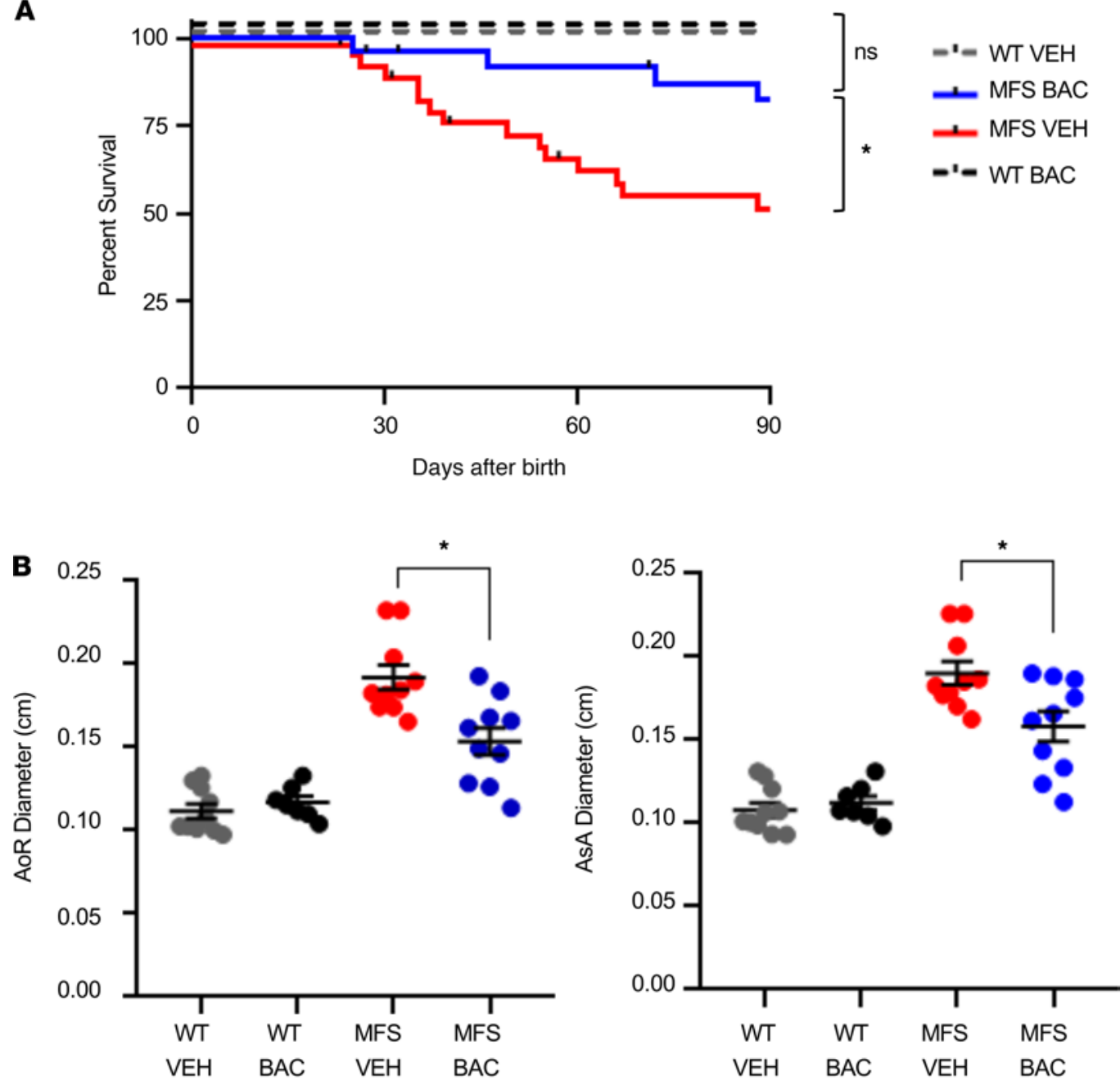

Figure 3. Improved median survival associated with alleviated arterial disease in baclofen-treated MFS mice. (A) Kaplan-Meier survival curves of WT and MFS mice treated with either baclofen (BAC) or vehicle (VEH). Number of mice analyzed in each treatment ranged from 20 (WT with VEH and BAC) to 25 (MFS with BAC) to 33 (MFS with VEH). The black marks in the survival curves of vehicle- and baclofen-treated mice signify animals that died from reasons other than ruptured TAA (i.e., pulmonary insufficiency or heart failure; ref. 16). ${ }^{*}$ Mantel-Cox test determined $P=0.0287$ significance among experimental groups, and Fisher's exact test determined $P=0.0078$ significance between vehicleand baclofen-treated MFS mice at P90. (B) Diameters of the aortic root (AoR; left) and proximal ascending aorta (AsA; right) in 3-month-old WT and MFS mice treated with either vehicle or the indicated drugs ( $n=7-10$ per each genotype and treatment). $2 \times 2$ Factorial ANOVA followed by Student's $t$ tests were employed to determine statistically significant differences $(P \leq 0.05)$, which are indicated by an asterisk. Data presented as mean \pm SD.

ically administered in drinking water to WT and mutant mice for 74 days starting at P16 and its impact on TAA progression was determined by monitoring the survival curve (and implicitly, the rate of aortic dissection and rupture) of MFS mice until P90 (when $\sim 50 \%$ of them are expected to be still alive; ref. 16). P90 was also the stage in which clinical, histopathological, biomechanical, and cellular surrogate parameters of arterial disease were examined. The relative levels of phosphorylated (p-) Smad2 and Erk1/2 (molecular surrogate parameters of TAA; refs. 6,7) were instead estimated at P60, a stage at which activation of both proteins is abnormally high in the dilating aorta of mice with progressively severe MFS (9).

We also conducted pharmacokinetics analyses to determine baclofen concentration in the plasma of treated mice. To that end, the drug was administered for 24 hours to eight 1-month-old WT mice that were sacrificed for plasma collection either immediately or 6 hours after completing the 24-hour treatment period ( $n=4$ per treatment group). HPLC/MS-MS based measurements of plasma baclofen in the first and second group of mice revealed mean drug concentrations of $271 \pm 61.04 \mathrm{ng} / \mathrm{ml}$ and $72.5 \pm 30.6 \mathrm{ng} / \mathrm{ml}$, respectively. The former value was comparable with the human $C_{\max }(211 \pm 42.8$ 
Table 1. Blood pressure measurements of mice of the indicated genotypes and treatments

\begin{tabular}{cccc}
\hline Mice & Systolic & Diastolic & Mean \\
WT + VEH & $105 \pm 6$ & $76 \pm 6$ & $85 \pm 6$ \\
WT + BAC & $84 \pm 5$ & $63 \pm 4$ & $70 \pm 6$ \\
MFS + VEH & $110 \pm 8$ & $77 \pm 7$ & $88 \pm 6$ \\
MFS + BAC & $82 \pm 6$ & $64 \pm 7$ & $70 \pm 6$ \\
MFS + PRO & $84 \pm 1$ & $63 \pm 2$ & $70 \pm 1$ \\
MFS + ENA & $81 \pm 3$ & $58 \pm 2$ & $66 \pm 1$
\end{tabular}

VEH, vehicle; BAC, baclofen; MFS, Marfan syndrome; PRO, propranolol; ENA, enalapril.

$\mathrm{ng} / \mathrm{ml}$ ) obtained at a low therapeutic dose of $10 \mathrm{mg}$ baclofen per day (18). We therefore concluded that the dose of baclofen used for our preclinical studies in mice is well within the FDA-approved dosage for both adult and pediatric subjects (20 to $80 \mathrm{mg}$ /day).

Systemic administration of baclofen led to a statistically significant extension of the median survival of MFS mice - and implicitly, a statistically significant delay of TAA dissection and rupture - as result of a substantial reduction in the rate of aneurysm growth relative to vehicle-treated animals (Figure 3, A and B). In line with hypotension being an infrequent side effect of baclofen administration to humans $(9 \%$ of cases as per https://www.rxlist.com/baclofen-drug.htm\#description), the drug treatment also lowered blood pressure in both WT and MFS mice (Table 1). However, similar to what has been previously reported for MFS mice with nondissecting TAA (19), the antihypertensive drugs propranolol and enalapril (both administered at hemodynamically equivalent doses to baclofen; Table 1) did not modify aneurysm growth and the rate of aortic dissection in MFS mice with lethal TAA (Supplemental Figure 4, A and B). This last finding therefore ruled out the possibility of a substantial protective effect of pharmacologically induced hypotension on aneurysm formation. Consistent with the phenotypic findings, postmortem examination of aortic wall histopathology of baclofen-treated MFS mice sacrificed at P90 revealed improved medial cellularity, nearly normalized elastic fiber morphology, and mitigated vessel wall fibrosis relative to vehicle-treated mutant animals (Figure 4). Furthermore, drug treatment was also associated with normal amounts of p-Smad2 and p-Erk1/2 in the aorta of MFS mice (Figure 5). Collectively, our analyses demonstrated that baclofen treatment can effectively delay TAA dissection and rupture in mice with progressively severe MFS.

Baclofen stimulates muscle contractility-related SCPs in MFS mice. To test if the therapeutic effect of baclofen was related to the SCPs used for its selection, we examined DEG profiles in the P60 aortas of baclofenversus vehicle-treated WT and MFS mice (Figure 6, A and B, and Supplemental Table 10). These analyses revealed that drug treatment upregulated genes associated with distinct SCPs related to muscle contractility and excitability in WT versus MFS aortas (Figure 6, C and D, and Supplemental Table 11). By contrast, analysis of downregulated genes did not predict any muscle-related SCPs (Supplemental Figure 4, A and B, and Supplemental Table 11). Taken together, these results suggest that $\mathrm{GABA}_{\mathrm{B}}$ agonism induces the same muscle responses by stimulating different subcellular mechanisms in WT versus MFS mice.

Experimental testing of our predictions showed that long-term baclofen treatment of MFS mice significantly increased the intracellular $\mathrm{Ca}^{2+}$ response of cultured primary aortic SMCs to the muscarinic receptor agonist carbachol (Figure 6E and ref. 20). Improved responsiveness to receptor-regulated calcium mobilization led us to hypothesize that normalized cellular muscle excitability would strengthen vascular tone, thus translating into improved mechanical compliance of the fibrillin-1-deficient aorta. Indeed, ex vivo biaxial mechanical testing of aortas from baclofen-treated MFS mice revealed a statistically significant improvement of vessel distensibility associated with a statistically significant reduction of circumferential wall stress and material stiffness compared with vehicle-treated MFS mice (Figure 7). Of note, we previously showed that increased circumferential wall stiffness is a strong indicator of aneurysmal propensity or presence (21). By contrast, no appreciable effects on elastic energy storage capacity were detected as a result of drug treatment (Figure 7). In line with the observation that baclofen lowers blood pressure in mice (Table 1), improved biomechanical values were most evident when calculated at the relevant systolic blood pressure (Figure 7 and Supplemental Table 12A) rather than at the common pressure of $100 \mathrm{mmHg}$ (Supplemental Figure 4 and Supplemental Table 12B). The finding that circumferential material stiffness was reduced under both systolic and common pressure was interpreted to 


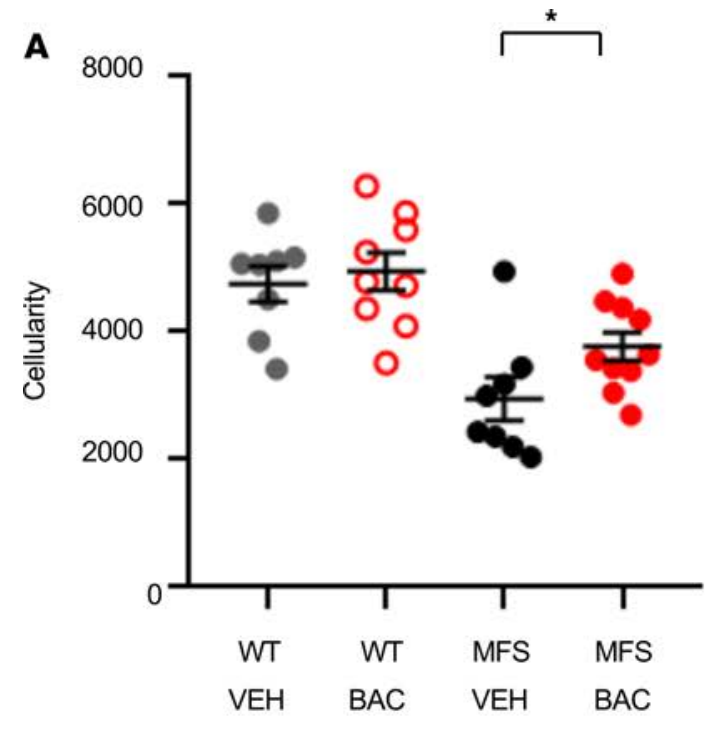

$H \& E$
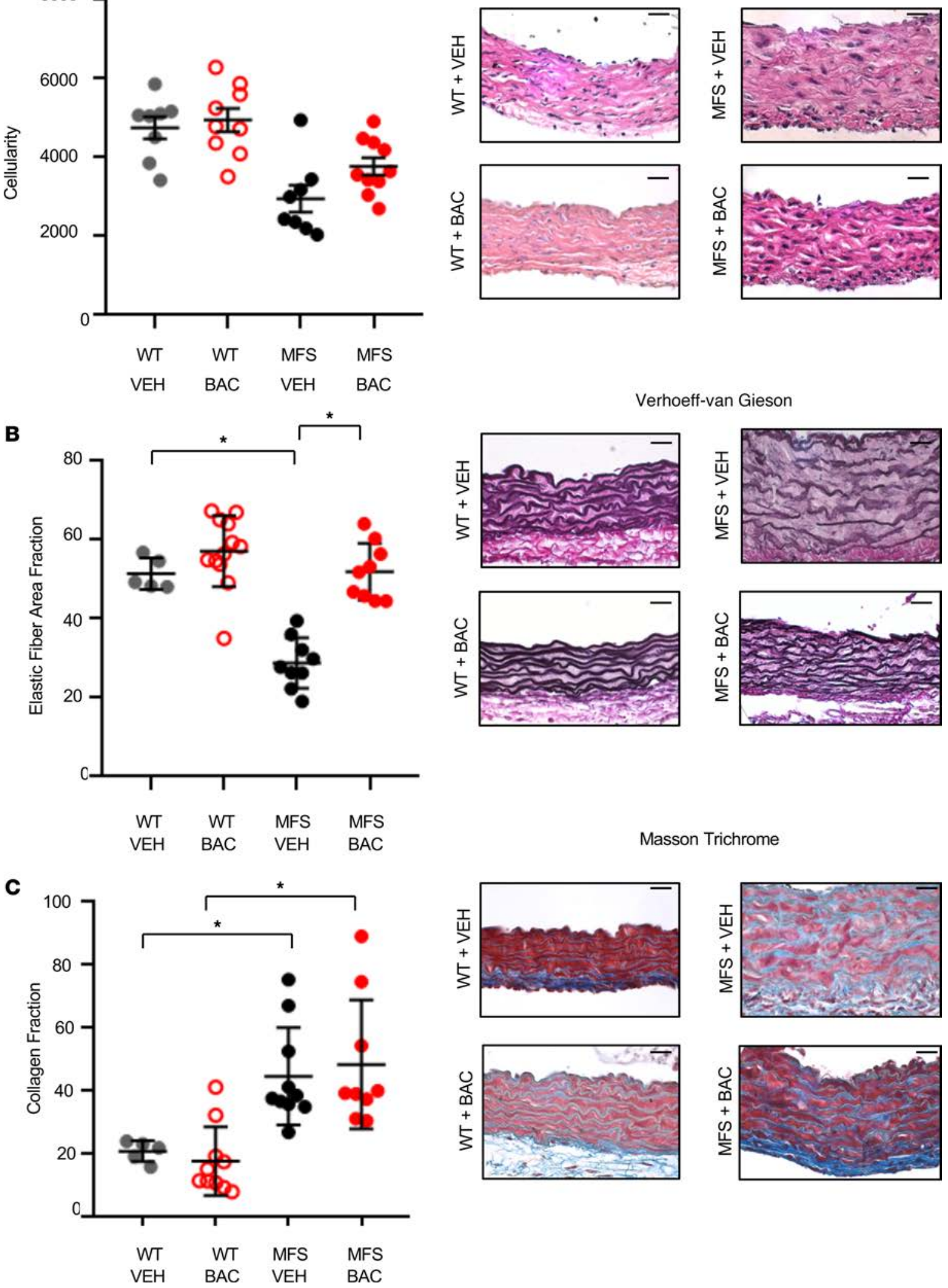

Figure 4. Mitigated TAA pathology in baclofen-treated MFS mice. Representative images of cross-sections of aortic walls of indicated genotype and treatment stained with (A) H\&E to estimate nuclei count, (B) Verhoeff-van Gieson stain to estimate elastic fiber architecture, and (C) Masson's trichrome to estimate collagen deposition. Data quantification is shown in the dot plots next to each of the panels ( $n=5-12$ per genotype and treatment group). 2 $\times 2$ Factorial ANOVA followed by Student's $t$ test were employed to determine statistically significant differences $(P \leq 0.05)$, which are indicated by an asterisk. Data presented as mean \pm SD. Scale bars: $25 \mu \mathrm{m}$. 
A

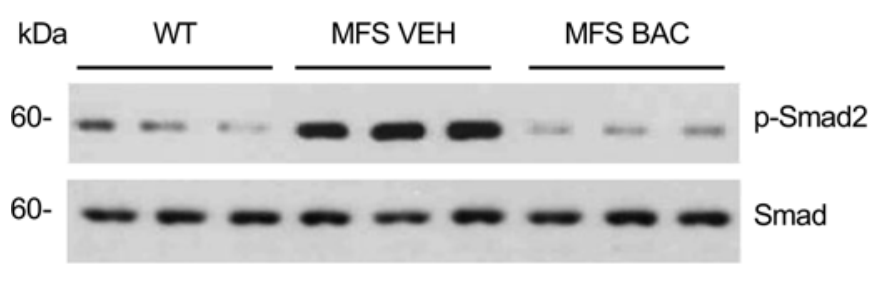

C

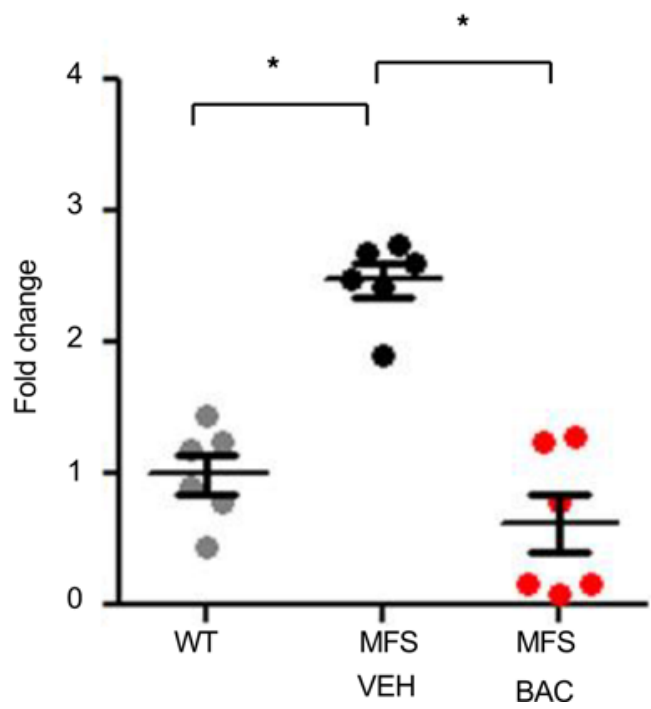

\section{B}

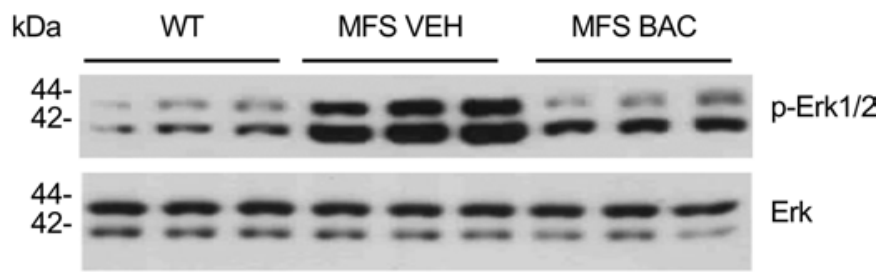

D

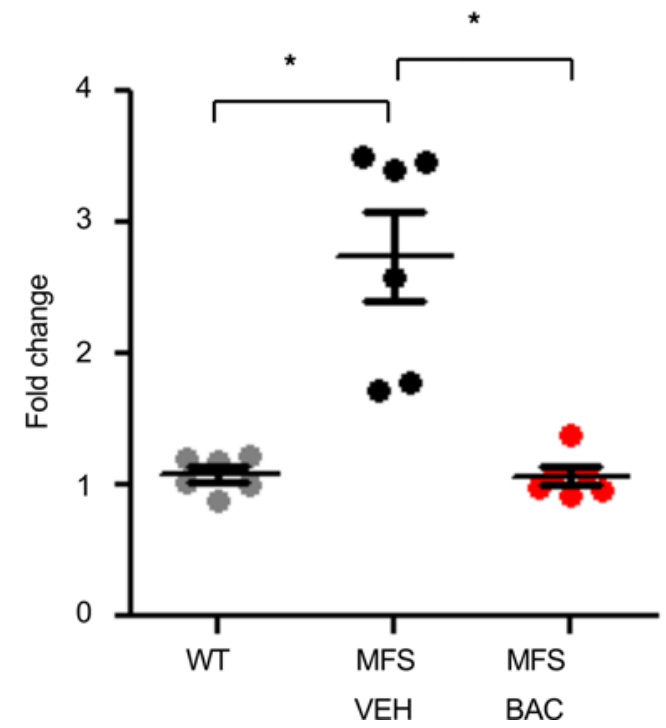

Figure 5. Normalized Smad and Erk activation in aortas of baclofen-treated MFS mice. Representative immunoblots of Smad2 (A) and Erk1/2 (B) proteins in the aortas of mice of the indicated genotypes and treatment with dot plots below them (C and $\mathbf{D})$ summarizing the fold change of phosphorylated versus total proteins ( $n=6$ per group). Average WT value is arbitrarily defined as 1 . Student's $t$ test was employed to determine statistically significant differences $(P \leq 0.05)$, which are indicated by an asterisk. Data presented as mean \pm SD.

strongly indicate that pressure alone could not modify this biomechanical property of the mutant aorta. Similar to MFS patients, these analyses also confirmed that the ascending aorta of MFS mice is intrinsically stiffer than the WT counterpart. Altogether, our data demonstrated that baclofen, an FDA-approved $\mathrm{GABA}_{\mathrm{B}}$ agonist, significantly extended the median survival of MFS mice prone to TAA dissection and rupture by improving aortic wall microarchitecture, contractility, and biomechanical function.

\section{Discussion}

It is hoped that pathologies that normally require surgical intervention, such as aortic aneurysms, would eventually be treated with drugs that, by slowing disease progression, reduce the complexity of pathology and comorbidity and allow stabilization and management of the abnormal phenotype. Among many others, a successful example of this approach is the treatment of hypertension, which if untreated often leads to myocardial infarctions or stroke, but with drug therapy can be managed for decades (22). Searching for a treatment that controls early determinants of pathophysiology would be particularly helpful, but inferring drug therapy from pathophysiological findings requires caution. Characterization of MFS mice with nondissecting TAA ( Fbn $1^{\mathrm{Cl039G/+}}$ mice) had originally concluded that aneurysm formation is due to a combination of AT1r-stimulated TGF- $\beta$ signaling and uncontrolled release of latent TGF- $\beta$ from a fibrillin-1-deficient ECM $(6,7)$. This disease mechanism was largely based on the finding that either AT1r antagonism by losartan or TGF- $\beta$ inhibition by a neutralizing antibody could prevent TAA formation. However, subsequent clinical trials with several large cohorts of MFS patients showed that the losartan neither mitigated the rate of aneurysm growth more than currently used beta blockers nor modified clinical endpoints of the disease (i.e., aortic dissection, elective aortic surgery, cardiovascular death) $(4,23)$. Exacerbated TAA pathology in $\mathrm{Fbn}^{\mathrm{Cl} 1039 \mathrm{G} / \mathrm{+}}$ mice with genetic disruption of TGF- $\beta$ signaling in postnatal SMCs further refuted the earlier postulate that TGF- $\beta$ is the primary trigger of TAA formation in MFS $(8,10)$. Indeed, a study of MFS mice with dissecting TAA (Fbn $1^{m g R / m g R}$ mice) 
A

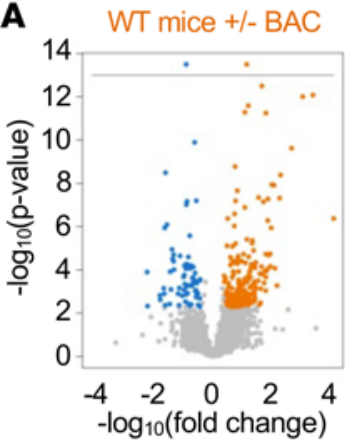

$66 \quad 185$

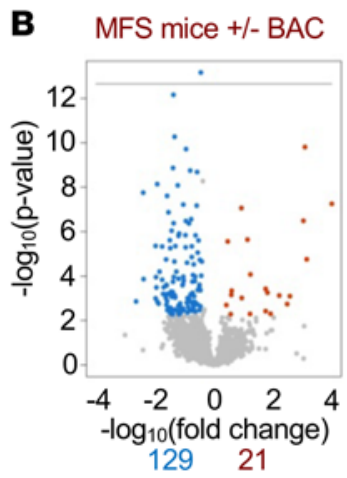

$129 \quad 21$
C

Upregulated SCPs in WT mice +/- BAC

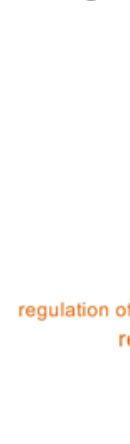

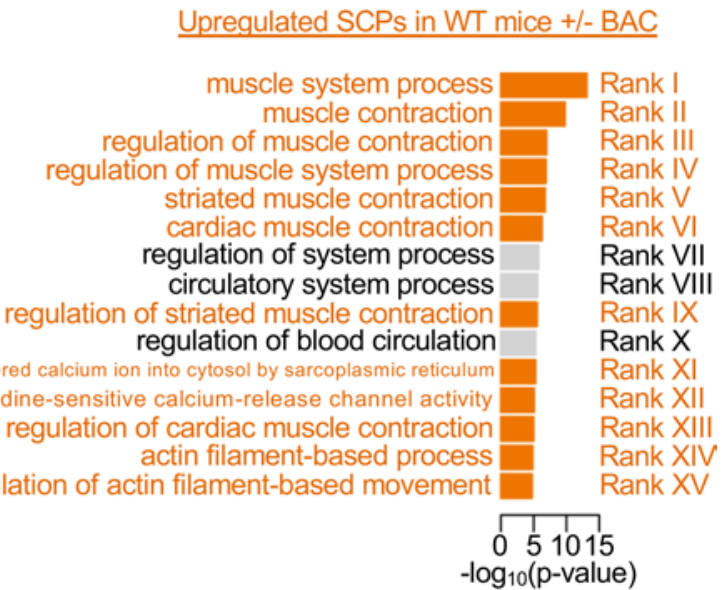

D

Upregulated SCPs in MFS mice $+/-$ BAC

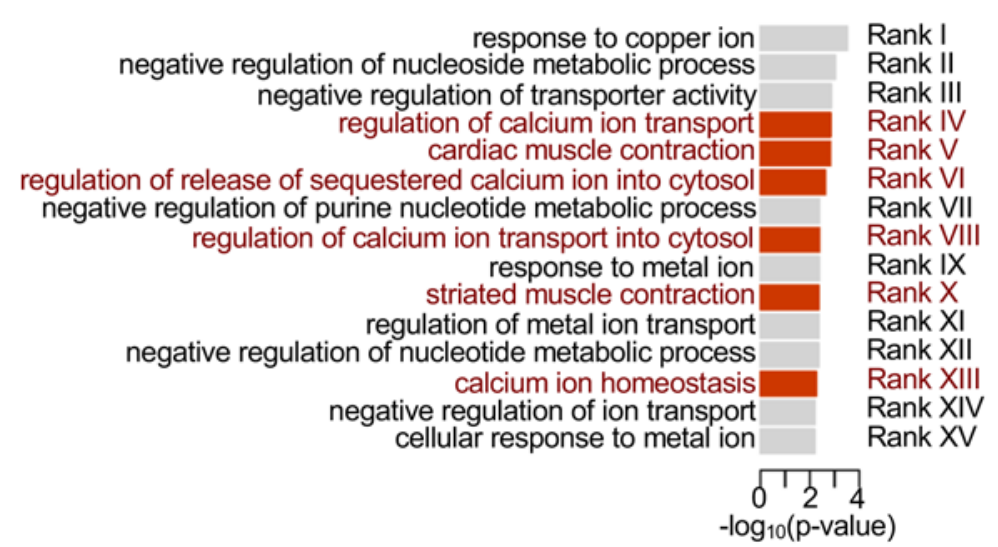

E 8

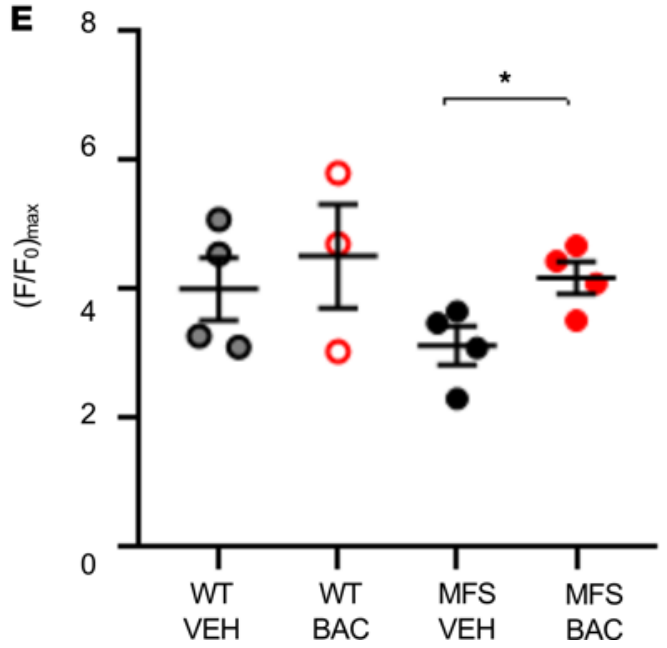

Figure 6. Baclofen treatment improves smooth muscle contractility and excitability in MFS mice. Volcano plots of DEGs in the aortas of WT (A) and MFS mice (B) sacrificed at the end of treatment with either baclofen or vehicle ( $n=2-4$ for each condition; for details see Figure 1A). Top-ranked SCPs identified in $\mathrm{CO}$ enrichment analysis of baclofen-upregulated genes in WT (C) and MFS mice (D) treated with either drug or vehicle (see also Supplemental Figure 3); WT and mutant muscle contractility-related processes are in orange and red, respectively. (E) Dot plot of mean peak heights of time traces acquired from single cells (and averaged per mouse) within 15 minutes of carbachol addition from 3 technical replicates ( $n=4$ for vehicle-treated WT and MFS mice, and $n=3$ and 4 for baclofen-treated WT and MFS cells, respectively). $2 \times 2$ Factorial ANOVA was used to determine statistically significant differences ( $P \leq$ $0.0524)$, which are indicated by an asterisk. Data presented as mean \pm SD.

also revealed opposite, stage-specific contributions of TGF- $\beta$ signaling to TAA development - i.e., protective during postnatal vessel growth and pathological later in arterial disease progression (9).

The disappointing outcome of the losartan clinical trials and the apparently dimorphic role of TGF- $\beta$ signaling during TAA progression have highlighted the inherent limitations of assessing complex pathophysiological processes by examining candidate signaling pathways in isolation. Integration of experimental and computational approaches to characterize disease-centered SCP networks represents a potentially powerful unbiased alternative to identify critical determinants of disease progression that could be targeted by pathwayand stage-specific drugs $(1,3)$. Using this strategy, we did not anticipate "correcting" the disease-associated transcriptome by searching for a drug that counter-regulates both upregulated and downregulated genes, as several different studies have done before (24-35). Rather, our approach sought to identify selective subcellular functions whose misregulation could be predicted to be involved in disease pathogenesis. We used the CMap database to identify drugs that affect the same subcellular functions without considering the identity of individual genes differentially expressed in MFS aortas. This approach has 2 advantages. By comparing expression profiles between afflicted patients and mutant mice, we can ensure that SCPs relevant to human diseases are targeted. By considering SCPs rather than individual DEGs, we can utilize disease mechanisms for drug discovery and thus raise the relevance of animal studies for human therapies. 
A

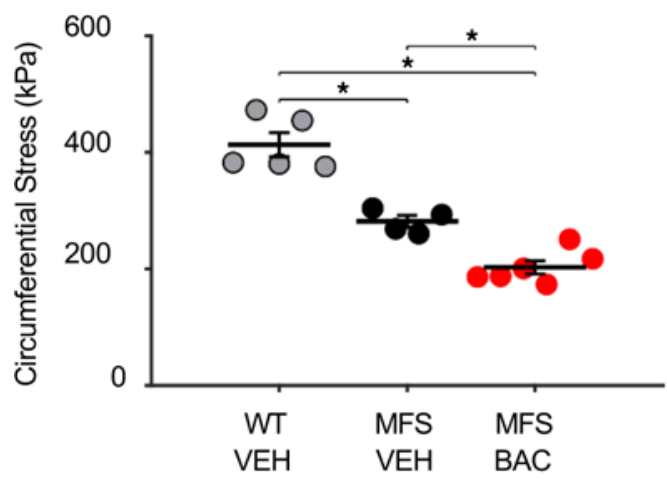

\section{C}

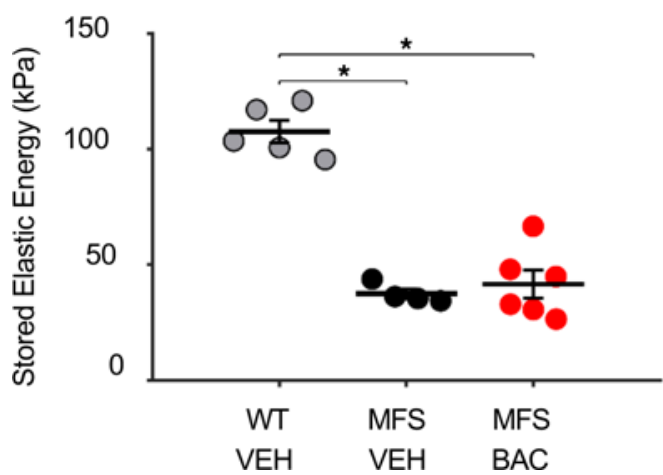

B

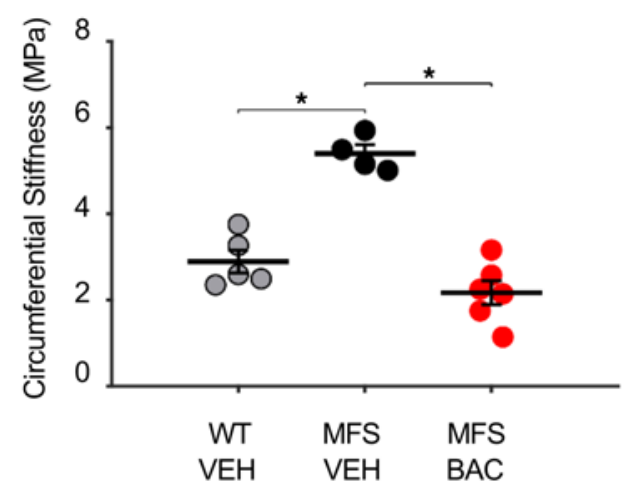

D

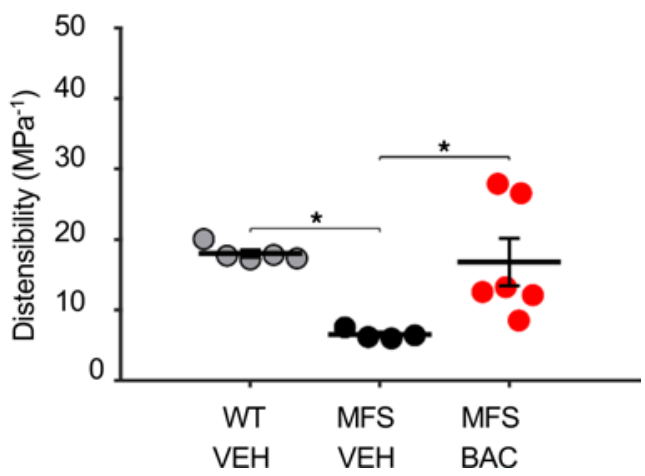

Figure 7. Baclofen treatment improves biomechanical function of the MFS mouse aorta. Dot plots summarizing the results from ex vivo biaxial mechanical tests of the ascending aorta of mice of the indicated genotype and treatment showing $(\mathbf{A})$ reduced circumferential wall stress, (B) normalized circumferential material stiffness, (C) unchanged elastic energy storage capability, and (D) improved aorta distensibility in baclofen- relative to vehicle-treated MFS mice. All values were calculated at the group-specific systolic pressure and in vivo value of the axial stretch $(n=4-6)$. Additional biomechanical data relevant to this figure are included in Supplemental Table $12 \mathrm{~A}$. Student's $t$ test was employed to determine statistically significant differences $(P \leq 0.05)$, which are indicated by an asterisk. Data presented as mean \pm SD.

Our results indicate that an integrative approach combining human and mouse transcriptomic data with prior knowledge of gene-SCP associations and disease pathophysiology allows drug repurposing even if the experimental data are based on relatively small sample sizes. The focus on SCPs (instead than individual genes) increases signal-to-noise ratios by eliminating "false-positive" DEGs from further analyses, as they usually belong to multiple different SCPs with low significance ranking. Additional increases in signal-tonoise ratios are accounted for by the exclusive focus on those convergent disease mechanisms that are independently predicted from both human and mouse data. Integration of computational predictions from whole genome expression profiles with prior knowledge of gene-SCP associations also increases our confidence in the predictions, as only disease-relevant mechanisms (i.e., SCPs) are further used in the study design.

Our work implicates $\mathrm{GABA}_{\mathrm{B}}$ signaling as an unsuspected modulator of TAA pathology in MFS. This finding is in agreement with previous in vitro experiments showing that $\mathrm{GABA}_{\mathrm{B}}$ receptors are expressed in human aortic SMCs where they transiently regulate intracellular $\mathrm{Ca}^{2+}$ concentration and presumably, muscle contractility (36), though we describe structural changes induced by long-term $\mathrm{GABA}_{\mathrm{B}}$ receptor stimulation. It should be noted that baclofen stimulation of SMC contractility is a new property of this drug distinct from the known muscle relaxing effect mediated through innervation (37). As the main scope of our study was to develop and validate a new computational strategy for drug repurposing, more detailed analyses of baclofen's mechanism of action on TAA progression and its probable impact on cardiac and endothelial dysfunction are the focus of ongoing investigations. Along the same lines, the use of aortic SMCs (as opposed to whole aortic tissue) might have limited the identification of other disease-related SCPs in common between human and mouse specimens. Future availability of both aortic SMCs and tissue from individual MFS patients will 
address this potential problem. Although they converge on SCPs related to muscle contractility, the identity of genes whose expression is altered by baclofen treatment is different than the identity of genes whose expression is associated with disease. Thus, the focus on SCPs indicative of dysregulated cell function allows for drug discovery beyond DEG identity. It is noteworthy that baclofen-induced mitigation of dissecting TAA is associated with improved microstructure (i.e., cellular) and biomechanical (i.e., physiological) function. We are therefore hopeful that our experimental findings will eventually be translated into clinical trials testing baclofen's ability to influence thoracic aortic disease progression in MFS patients.

\section{Methods}

Animals and drug treatments. All experiments used male Fbn1 ${ }^{m g R / m g R}$ (MFS) mice and sex-and age-matched WT littermates maintained on the C57BL/6J genetic background (16). Male animals ensured consistency with previous work using the same mouse model of MFS by avoiding sex-related differences in TAA progression $(9,13)$. Baclofen (Sigma Life Science), enalapril, or propranolol (LKT Labs) was administered to mice in drinking water from P16 until P90. Drug concentrations were $0.15 \mathrm{~g} / 1$ (enalapril) and $0.5 \mathrm{~g} / 1$ (propranolol) per body mass; baclofen concentration was either $0.1 \mathrm{~g} / 1$ or $0.2 \mathrm{~g} / 1$ per body mass, depending on whether the mice weighed less or more than 10 grams, respectively. Plasma was collected from 30-dayold WT mice treated with baclofen for 24 hours and sacrificed by cardiac puncture either immediately after baclofen withdraw (4 mice) or 6 hours later (4 mice). Sample preparation including calibration curves, quality controls, LC-MS/MS, and data analysis were performed as previously described (38, 39). Briefly, the liquid chromatographic/triple-quadrupole (LC-MS/MS) system consisted of a Shimadzu Prominence HPLC system connected to a Sciex API 3000 triple-quadrupole instrument equipped with electrospray ionization (ESI) source. The data acquisition was under the control of Analyst software (version 1.4.2). Baclofen and the baclofen-D4 internal standard (IS) were separated on a Phenomenex C18 column ( $2 \mathrm{~mm}$ $\times 50 \mathrm{~mm}, 4 \mu \mathrm{m}$ ). The initial mobile phase consisted of $0.1 \%$ formic acid (A) and acetonitrile with $0.1 \%$ formic acid (B) with gradient elution at a flow rate of $0.2 \mathrm{ml} / \mathrm{min}$ and an injection volume of $3 \mu \mathrm{l}$. The gradient program was ( $\mathrm{min} / \% \mathrm{~B}): 0.2,5 / 95,8 / 95.8 .10 / 2,12 / 2$. The multiple-reaction-monitoring modes of ions with $m / z 214.3 \rightarrow 151.2$ for baclofen, and $m / z 218.30 \rightarrow 155.20$ for IS were utilized for quantitative analysis. Regression analyses of peak area ratios of baclofen/IS versus baclofen concentrations were used to assess linearity utilizing $1 / \chi$ weighting factor $\left(\chi=\right.$ concentration). The calibration curves were linear $\left(r^{2}\right.$ $>0.998$ ) over the baclofen concentration range of $1-2500 \mathrm{ng} / \mathrm{ml}$ in plasma. Based on analyzing quality control samples in triplicate, the intraday and interday precision $(\% \mathrm{CV})$ and the accuracy were equal to or less than $\pm 11 \%$ of the nominal concentrations. The recovery of baclofen and IS in plasma was greater than $90 \%$. Mouse median survival was calculated from weaning to P90 when surviving mice were sacrificed for tissue collection. Censored events due to death other than dissecting TAA were recorded and their time included in the survival curve. TAA progression in MFS mice was monitored by ultrasound (VisualSonics Vevo 2100 imaging system and a $40-\mathrm{MHz}$ transducer) using long-axis parasternal views of the aorta in mice under isoflurane anesthesia $(6,9,13)$. B-mode 2-dimensional videos were captured and used to measure aortic root and ascending aorta diameter with the aid of VisualSonics software. Measurements were taken in systole from 3-4 images per mouse by a single individual blinded to genotype and treatment, and values were averaged to obtain diameter per mouse $(6,9,13)$. Tail-cuff blood pressure measurements were performed on each group of 3-month-old conscious mice using the CODA noninvasive blood pressure system (Kent Scientific). Mice were habituated to the tail-cuff pressure system over the course of 2 days with subsequent recording over the course of 3 days (40). The average systolic, diastolic, and pulse pressure measured in more than 30 blood pressure recordings was used for each animal ( $n=4-5$ per group).

Patients and aortic SMC cultures. Three male MFS patients who underwent aortic root surgery (patients FB359, FB490, and FB721) were recruited through the National Reference Center for MFS and Related Disorders (Hôpital Bichat) and diagnosed according to established clinical criteria at the age of 22 (FB359), 29 (FB490), and 4 years (FB721) (41). Patient FB359 [FBN1 mutation: c.4786C>T - p.(Arg1596*)] underwent prophylactic surgery at the age of 32 because of both progressing aortic dilatation $(48 \mathrm{~mm})$ despite beta blocker therapy, and massive mitral regurgitation secondary to mitral valve prolapse. He had no opthalmologic feature except flat cornea. He was tall $(195 \mathrm{~cm})$, with pectus carinatum, scoliosis, hindfoot deformity, no wrist or thumb sign, no dural ectasia, protrusio acetabuli, increased arm span/height ratio, no scoliosis, facial features, and skin striae. Patient FB490 [FBN1 mutation: c. 4937G>A - p.(Cys1646Tyr)] underwent surgery at the age of 29 when progressive aortic dilatation was discovered $(53 \mathrm{~mm})$ without mitral valve prolapse. He presented 
wrist and thumb signs, slight pectus carinatum deformity, no hindfoot deformity, no pneumothorax, reduced elbow extension, mild scoliosis, and facial features; this patient had surgery for ectopia lentis during infancy. Patient FB721 [FBN1 mutation: c.4349_4351del - p.(Cys1450del)] underwent surgery at the age of 22 for progressive root aortic dilatation. He also presented mild mitral valve prolapse with minor regurgitation, wrist and thumb sign, pectus excavatum, hindfoot deformity, minor scoliosis, skin striae, facial features, and ectopia lentis was present since early infancy. TAA specimens from the outer curvature (the most dilated part of the ascending aorta) were collected during elective aortic surgery. Normal ascending aortas were obtained from 3 organ transplant donors (AS1043, AS1883, and AS1960) aged 22, 21, and 31 years, respectively. Mean age at the time of aortic tissue collection was 28 years for MFS patients and 25 years for non-MFS individuals. Aortic tissues were processed to separate medial and adventitial layers. Media samples were incubated in collagenase and elastase $0.1 \%$ ( 3 hours, $37^{\circ} \mathrm{C}$ ) to obtain aortic SMCs that were cultured in specific medium (Promocell) containing $20 \%$ fetal calf serum (FCS), insulin $(5 \mu \mathrm{g} / \mathrm{ml})$, human epidermal and fibroblast growth factors $(0.5$ and $2 \mathrm{ng} / \mathrm{ml}$, respectively), plasmocin $(2.5 \mu \mathrm{g} / \mathrm{ml})$, and primocin $(100 \mu \mathrm{g} / \mathrm{ml})$. Cells at passages 3-4 were used for RNA extraction performed after 24-hour incubation in serum-free medium.

$R N A$ purification and sequencing. Total RNA was extracted from human SMC cultures with Trizol Reagent (Life Technologies) and purified by miRNeasy Mini kit (Qiagen S.A.) according to the manufacturer's instructions. DNase digestion procedure was followed for each sample. RNA concentration was estimated by measuring absorbance at $260 \mathrm{~nm}$. RNA quality was assessed by agarose electrophoresis. Total RNA was purified from frozen mouse aortic tissues ( $n=3$ for MFS mice at P16, $n=3$ for WT mice at P16, $n=4$ for baclofen-treated WT mice at P60, $n=2$ for vehicle-treated WT mice at P60, $n=4$ for baclofen-treated MFS mice at P60, $n=$ 3 for vehicle-treated MFS mice at P60) using the RNeasy Fibrous Tissue Kit (Qiagen S.A.). RNA amounts were quantitated by Qubit fluorometer (Life Technologies) and RNA quality was assessed using an Agilent 2100 Bioanalyzer. Libraries were prepared from $1 \mu \mathrm{g}$ of total RNA using TruSeq Stranded mRNA kit protocol (Illumina) according to the supplier's recommendations. Briefly, poly-A RNA was isolated using oligo-dT $\mathrm{d}_{25}$ attached to magnetic beads, fragmented into approximately 300-bp-long pieces using divalent cations under elevated temperature, used as template for double-stranded cDNA synthesis, Illumina adapters ligation, and PCR-mediated cDNA library amplification. Sequencing of random paired-end 75-bp or single-ended 100-bp molecules was carried out on an Illumina HiSeq 4000 for human and mouse samples, respectively.

Identification of human and mouse DEGs. Single- and paired-end sequencing reads were aligned to the human reference genome 'hg19' or mouse reference genome 'mm9' using Tophat 2.0.8., samtools-0.1.7, and bowtie 2.1.0. (42). DEGs were identified with cufflinks 1.3.0 (43). All human samples and mouse samples at P16 and P60 were considered as 3 separate sets and DEGs were identified between experimental and control samples in each of them. To prevent the identification of false-positive DEGs due to read imbalances, sequencing reads were randomly removed from each sample of the same set before read alignment to ensure that each sample in that set had the same total read counts (44). Tophat was used with the mouse/human ensemble GTF file as a gene annotation reference and the option 'no-novel-juncs'. For the alignment of the human paired-end sequencing reads we specified '--read-align-edit-dist 0'. The inner mate distance was set to 311 bps (AS1043), 298 bps (AS1883), 273 bps (AS1960), 321 bps (FB359), 291 bps (FB490), and 285 bps (FB721). The mate-std-dev was set to 50 bps. Output BAM files were directly subjected to differential gene expression identification using Cufflinks with the options 'multi-read-correct,' 'upper-quartile-norm,' and 'frag-bias-correct' against the mm9/hg19 genome. DEGs were identified based on an FDR of 10\% and a minimum absolute fold change $\left(\log _{2}[(\right.$ FPKMcondition $1+1) /($ FPKMcondition $\left.2+1)]\right)$ of $\log _{2}(1.3)$.

CMap database and pathway enrichment analyses. The libraries "Old_CMAP_up" and "Old_CMAP_ down" were downloaded from the EnrichR website $(45,46)$. Lists of upregulated and downregulated genes either in the CMap database or experimentally generated were subjected to pathway enrichment analysis using Fisher's exact test and the "GO_Biological_Process_2015" library downloaded from the EnrichR website, as described previously (39). Background genes for the analysis of mouse/human DEGs were intersecting genes between all genes in the GO library and all genes that are annotated in the ensembl GTF file of the mm9/hg19 reference genome. Background genes for the analysis of the CMAP database were all intersecting genes between all genes in the GO library and all genes in the merged "Old_CMAP_up" and "Old_CMAP_down" libraries. For statistical accuracy, we removed all genes that were not part of the background set from DEGs or SCPs before performing the enrichment analysis and identifying disease-relevant SCPs. To identify drug candidates, we considered only SCPs for the further analysis that were among the top 15 predictions (ranked by $P$ value) and contained at least 20 genes. 
GO graph extension. GO Open Biomedical Ontologies was downloaded from the website http://geneontology.org/page/download-ontology, "go.obo." GO hierarchical network was generated based on the "is_a," "part_of," "regulates," "positively_regulates," "negatively_regulates” relationships. Regulatory relationships were inverted - i.e., source and target processes were switched. Disease-relevant SCPs were used as seed nodes in the GO graph. All ancestor and offspring processes of the seed-node processes were identified. SCPs that were annotated to less than 20 genes in the "GO_Biological_Process_2015" library or not part of it were removed. General and SMC-specific muscle contractility processes as well as processes involving actin-related processes were expertly curated from the generated GO subgraph. We also removed processes that described positive or negative regulatory functions (but not their parent processes with [unsigned] regulatory functions), if such removal did not split the GO subnetwork into multiple unconnected graphs.

Drug target genes identification. Human genes targeted by drugs were identified based on Drug Bank data (http://www.drugbank.ca). Any drug not in the CMap database or without known targeted human genes was excluded from further analysis. Mouse homologs were identified for the human targeted genes based on the Mouse Genome Informatics (MGI) (http://www.informatics.jax.org) and the National Center for Biotechnology Information (http://www.ncbi.nlm.nih.gov/homologene/database). All data processing steps after the identification of DEGs were coded in C\#. R was used to generate diagrams, yED was used to visualize networks.

Histological analyses. Aortas harvested from WT and MFS mice were fixed overnight in 10\% formalin followed by paraffin embedding and sectioning at $5 \mu \mathrm{m}$. Sections were stained with hematoxylin and eosin (H\&E), Masson's trichrome, or Verhoeff-van Gieson stain. Images were acquired using a Leica microscope (Model DM2500) with a $\times 40$ objective and analyzed using ImageJ $(\mathrm{NIH})$. For tissue cellularity (H\&E staining), nuclei were counted and normalized to area $(6,9,13)$. The density of elastic (Verhoeff-van Gieson staining) and collagen fibers (Masson's trichrome staining) were quantified using the ImageJ analysis software color threshold tool to define the area fraction of elastic fibers or collagen, and the ratio of their respective densities to the surface of the tunica media area was calculated (13). All histological analyses were independently performed by 2 individuals blinded to genotype and treatment. Three serial sections per mouse were used for each histological assessment in which 3 images of each individual section were analyzed and averaged $(6,9,13)$.

Intracellular calcium measurement. SMCs were isolated by collagenase digestion from the aortas of vehicle- or baclofen-treated WT and MFS mice $(n=3-4$ per genotype and treatment). Approximately 10,000 cells were seeded on 96-well black assay plates with clear, tissue culture-treated polystyrene bottoms (Corning). Intracellular $\mathrm{Ca}^{2+}$ measurements were performed as previously described $(12,47)$. Briefly, cells were labeled for 1 hour with calcium fluorophore Fluo4-AM (Invitrogen) 48 hours after seeding. For each experiment, the fluorophore was prepared fresh in Live Cell Imaging Solution (Molecular Probes, Life Technologies) to be used at a final concentration of $2.5 \mu \mathrm{M}$. Before measurements, cells were washed with Live Cell Imaging Solution and subsequently assayed using a Zeiss LSM 880 confocal microscope and a $\times 10$ objective, with $\mathrm{CO}_{2}$ and temperature control set at $5 \%$ and $37^{\circ} \mathrm{C}$, respectively. Fluorescence intensity was measured before and after addition of $100 \mu \mathrm{M}$ carbachol (Sigma-Aldrich) for a total of 15 minutes, with an acquisition rate of 1 frame/sec. For each well assayed, a field containing at least 10 cells was randomly picked and the temporal fluorescence intensities were extracted for responding cells using ZEN Software. A cell response was defined as having more than $10 \%$ increase in intensity upon stimulation compared with basal levels $\left(F / F_{0} \geq 1.10\right)$. Normalization of fluorescence intensities $\left(F / F_{0}\right)$ within 60 seconds after agonist addition was performed in MATLAB. Data of mice from the same group were averaged and results from different experimental groups were plotted in Prism as dot plots with mean and \pm SD.

Biomechanics. For ex vivo assessment of biaxial mechanical properties, following euthanasia the ascending aorta (from the root to brachiocephalic) and proximal descending thoracic aorta (from the left subclavian to the sixth pair of intercostals) were excised, cleaned of excess perivascular tissue, cannulated on glass micropipets, and placed within a custom computer-controlled testing device in a Hanks buffered physiologic solution maintained at $37^{\circ} \mathrm{C}$. Material behaviors were quantified using a validated constitutive relation based on cyclic pressure-diameter and axial force-length tests as described previously (48). Aorta distensibility was calculated as (systolic diameter - diastolic diameter)/(diastolic diameter $\times$ pulse pressure).

Immunoblotting. Proteins were extracted from frozen aortas and processed for immunoblots using antibodies against phosphorylated and nonphosphorylated Smad2 and Erk1/2 proteins (Cell Signaling Technology; refs. 9, 13). Antibodies against p-Erk1/2 (T202/Y204; catalog 4370) were diluted 1:2000, and antibodies against p-Smad2 (S465/467; catalog 3108), Erk1/2 (catalog 9102), and Smad2 (catalog 3102) were diluted 1:1000 before being used as per manufacturer's recommendations. Antibodies were diluted in 
Tris-buffered saline, $\mathrm{pH} 7.4$, and $0.1 \%$ (v/v) Tween 20 in the presence of 5\% nonfat milk and incubated with the transfer membrane for 16 hours at $4^{\circ} \mathrm{C}$. In all cases, ponceau S solution staining (Sigma-Aldrich) of the transfer membrane was used as a protein loading control. Immunoreactive products were visualized by chemiluminescence using Amersham ECL Select Western Blotting Detection Reagent (GE Healthcare) and their relative intensity was evaluated using Photoshop (Adobe Systems Inc.).

Statistics. The nonparametric Mantel-Cox test was used to analyze survival curves. To compare the mean differences between groups (vehicle- and baclofen-treated) that have been split between 2 independent variables (WT and MFS), a $2 \times 2$ factorial analysis of variance (ANOVA) design was used. This design tests both experimental factors (drug and genotype) simultaneously and also tests for a possible interaction between the 2 factors. For significant interaction terms, individual $t$ tests were performed for each group (baclofen- or vehicle-treated) per genotype (WT or MFS). All values were expressed as the mean $\pm \mathrm{SD}$. $P$ values were not adjusted for the multiple tests that were performed, protecting against the inflation of the Type I error. Analyses were performed using SAS System Software, version 9.4 and GraphPad Prism version 7.0.

Study approval. The Institutional Animal Care and Use Committees of the Icahn School of Medicine at Mount Sinai in New York City reviewed and approved all animal studies. The clinical research protocol was approved by the local ethical committee (CPP 0504 32, Ambroise Paré, Boulogne, France, April 2005; updated in March 2008). Aortic specimens were collected from MFS patients during elective aortic surgery (Hôpital Bichat) and used in compliance with French regulations (Declaration of Conservation DC-2008283). Ascending aortas from organ transplant donors were obtained with the authorization of the French Biomedicine Agency (PFS 09-007) and in accordance with the Declaration of Helsinki. Informed consent was provided for all patients in agreement with the requirements of French bioethics laws.

Data and materials availability. RNA-Seq data and source code can be downloaded from http://www. sbcny.org and GEO GSE128101.

\section{Author contributions}

JH designed and performed all computational analyses. JG and CIC examined drug effects on TAA pathology in MFS mice and prepared relevant biological samples for ex vivo and in vitro analyses. JMG characterized drug pharmacokinetics with support from RB. MBM assisted with mouse work. PA, GJ, and CB collected clinical data and processed human samples. RCC and EUA measured intracellular calcium. BS, SIM, and JDH performed and interpreted biomechanical tests. AW performed statistical analysis. FR and RI conceived the study and wrote the manuscript with critical input from all authors.

\section{Acknowledgments}

We thank A. Stern for guidance with the intracellular $\mathrm{Ca}^{2+}$ measurements; I. Miramontes and M. Yu for assistance with the mouse studies; G. Jayaraman for assistance with cell culture of the primary mouse cells; and K. Johnson for organizing the manuscript. This work was supported by NIH grants AR069307 (to FR and RI), HL134605 (to FR, RI, and JH) and GM071558 and GM072853 (to RI), by the Agence Nationale de la Recherche grant NONAGES-14CE15-0012, the Fédération Française de Cardiologie-2015 and a Bourse de la Fondation de Cardiologie-2016 (to GJ), by the Netherlands Organization for Scientific Research (NWO) Rubicon grant 452172006 (to BS), by SUNY at Buffalo start-up funds (to JG), and by the National Marfan Foundation and the Elster Research Endowment (to FR).

Address correspondence to: Ravi Iyengar, Icahn School of Medicine at Mount Sinai, 1425 Madison Avenue, Box 1215, New York, New York 10029. Phone: 212.659.1717; Email: ravi.iyengar@mssm.edu. Or to: Francesco Ramirez, Icahn School of Medicine at Mount Sinai, 1468 Madison Avenue, Box 1603, New York, New York 10029. Phone: 212.241.2238; Email: francesco.ramirez@mssm.edu.

\footnotetext{
1. Hansen J, Iyengar R. Computation as the mechanistic bridge between precision medicine and systems therapeutics. Clin Pharmacol Ther. 2013;93(1):117-128.

2. Baudino TA. Targeted cancer therapy: the next generation of cancer treatment. Curr Drug Discov Technol. 2015;12(1):3-20.

3. Zhao S, Iyengar R. Systems pharmacology: network analysis to identify multiscale mechanisms of drug action. Annu Rev Pharmacol Toxicol. 2012;52:505-521.

4. Milewicz DM, Prakash SK, Ramirez F. Therapeutics targeting drivers of thoracic aortic aneurysms and acute aortic dissections: insights from predisposing genes and mouse models. Annu Rev Med. 2017;68:51-67.

5. Ramirez F, Caescu C, Wondimu E, Galatioto J. Marfan syndrome; A connective tissue disease at the crossroads of mechano-
} 
transduction, TGF $\beta$ signaling and cell stemness. Matrix Biol. 2018;71-72:82-89.

6. Habashi JP, et al. Losartan, an AT1 antagonist, prevents aortic aneurysm in a mouse model of Marfan syndrome. Science. 2006;312(5770):117-121.

7. Holm TM, et al. Noncanonical TGF $\beta$ signaling contributes to aortic aneurysm progression in Marfan syndrome mice. Science. 2011;332(6027):358-361.

8. Li W, et al. Tgfbr2 disruption in postnatal smooth muscle impairs aortic wall homeostasis. J Clin Invest. 2014;124(2):755-767.

9. Cook JR, et al. Dimorphic effects of transforming growth factor- $\beta$ signaling during aortic aneurysm progression in mice sugges a combinatorial therapy for Marfan syndrome. Arterioscler Thromb Vasc Biol. 2015;35(4):911-917.

10. Wei $\mathrm{H}$, et al. Aortopathy in a mouse model of Marfan syndrome is not mediated by altered transforming growth factor $\beta$ signaling. J Am Heart Assoc. 2017;6(1):e004968

11. Oller J, et al. Nitric oxide mediates aortic disease in mice deficient in the metalloprotease Adamts1 and in a mouse model of Marfan syndrome. Nat Med. 2017;23(2):200-212.

12. Granata A, et al. An iPSC-derived vascular model of Marfan syndrome identifies key mediators of smooth muscle cell death. Nat Genet. 2017;49(1):97-109.

13. Galatioto J, et al. Cell type-specific contributions of the angiotensin II type 1a receptor to aorta homeostasis and aneurysma disease-brief report. Arterioscler Thromb Vasc Biol. 2018;38(3):588-591.

14. Milewicz DM, Ramirez F. Therapies for thoracic aortic aneurysms and acute aortic dissections. Arterioscler Thromb Vasc Biol. 2019;39(2):126-136.

15. Lamb J, et al. The Connectivity Map: using gene-expression signatures to connect small molecules, genes, and disease. Science 2006;313(5795):1929-1935.

16. Pereira L, et al. Pathogenetic sequence for aneurysm revealed in mice underexpressing fibrillin-1. Proc Natl Acad Sci USA. 1999;96(7):3819-3823.

17. Lino Cardenas CL, et al. Inhibition of the methyltranferase EZH2 improves aortic performance in experimental thoracic aortic aneurysm. JCI Insight. 2018;3(5):97493.

18. Shellenberger MK, Groves L, Shah J, Novack GD. A controlled pharmacokinetic evaluation of tizanidine and baclofen at steady state. Drug Metab Dispos. 1999;27(2):201-204.

19. Habashi JP, et al. Angiotensin II type 2 receptor signaling attenuates aortic aneurysm in mice through ERK antagonism. Science. 2011;332(6027):361-365.

20. Dolphin AC, Scott RH. Calcium channel currents and their inhibition by (-)-baclofen in rat sensory neurones: modulation by guanine nucleotides. J Physiol (Lond). 1987;386:1-17.

21. Bellini C, et al. Comparison of 10 murine models reveals a distinct biomechanical phenotype in thoracic aortic aneurysms. $J R$ Soc Interface. 2017;14(130):20161036.

22. Oparil S, Schmieder RE. New approaches in the treatment of hypertension. Circ Res. 2015;116(6):1074-1095.

23. De Backer J. Marfan and Sartans: time to wake up! Eur Heart J. 2015;36(32):2131-2133.

24. Boyle JO, et al. Effects of cigarette smoke on the human oral mucosal transcriptome. Cancer Prev Res (Phila). 2010;3(3):266-278.

25. Chang M, Smith S, Thorpe A, Barratt MJ, Karim F. Evaluation of phenoxybenzamine in the CFA model of pain following gene expression studies and connectivity mapping. Mol Pain. 2010;6:56.

26. Chen B, Butte AJ. Leveraging big data to transform target selection and drug discovery. Clin Pharmacol Ther. 2016;99(3):285-297.

27. Chen B, et al. Reversal of cancer gene expression correlates with drug efficacy and reveals therapeutic targets. Nat Commun. 2017;8:16022.

28. Dudley JT, et al. Computational repositioning of the anticonvulsant topiramate for inflammatory bowel disease. Sci Transl Med. 2011;3(96):96ra76.

29. Jahchan NS, et al. A drug repositioning approach identifies tricyclic antidepressants as inhibitors of small cell lung cancer and other neuroendocrine tumors. Cancer Discov. 2013;3(12):1364-1377.

30. Josset L, et al. Gene expression signature-based screening identifies new broadly effective influenza a antivirals. PLoS One. 2010;5(10):e13169.

31. Kunkel SD, et al. mRNA expression signatures of human skeletal muscle atrophy identify a natural compound that increases muscle mass. Cell Metab. 2011;13(6):627-638.

32. Pacini C, et al. DvD: An R/Cytoscape pipeline for drug repurposing using public repositories of gene expression data. Bioinformatics. 2013;29(1):132-134.

33. Sirota $\mathrm{M}$, et al. Discovery and preclinical validation of drug indications using compendia of public gene expression data. $S c i$ Transl Med. 2011;3(96):96ra77.

34. Wagner A, et al. Drugs that reverse disease transcriptomic signatures are more effective in a mouse model of dyslipidemia. Mol Syst Biol. 2015;11(3):791.

35. Zhong Y, et al. Renoprotective effect of combined inhibition of angiotensin-converting enzyme and histone deacetylase. $J A m$ Soc Nephrol. 2013;24(5):801-811.

36. Wang XP, Cheng ZY, Schmid KL. GABAB receptors expressed in human aortic endothelial cells mediate intracellular calcium concentration regulation and endothelial nitric oxide synthase translocation. Biomed Res Int. 2014;2014:871735.

37. Lapeyre E, Kuks JB, Meijler WJ. Spasticity: revisiting the role and the individual value of several pharmacological treatments NeuroRehabilitation. 2010;27(2):193-200.

38. Lv H, Zhang X, Sharma J, Reddy MV, Reddy EP, Gallo JM. Integrated pharmacokinetic-driven approach to screen candidate anticancer drugs for brain tumor chemotherapy. AAPS J. 2013;15(1):250-257.

39. Zhang X, et al. Preclinical pharmacological evaluation of a novel multiple kinase inhibitor, ON123300, in brain tumor models. Mol Cancer Ther. 2014;13(5):1105-1116.

40. Cook JR, et al. Abnormal muscle mechanosignaling triggers cardiomyopathy in mice with Marfan syndrome. J Clin Invest. 2014;124(3):1329-1339.

41. Loeys BL, et al. The revised Ghent nosology for the Marfan syndrome. JMed Genet. 2010;47(7):476-485.

42. Kim D, Pertea G, Trapnell C, Pimentel H, Kelley R, Salzberg SL. TopHat2: accurate alignment of transcriptomes in the pres- 
ence of insertions, deletions and gene fusions. Genome Biol. 2013;14(4):R36.

43. Trapnell C, et al. Transcript assembly and quantification by RNA-Seq reveals unannotated transcripts and isoform switching during cell differentiation. Nat Biotechnol. 2010;28(5):511-515.

44. Stillitano F, et al. Modeling susceptibility to drug-induced long QT with a panel of subject-specific induced pluripotent stem cells. Elife. 2017;6:e19406.

45. Chen EY, et al. Enrichr: interactive and collaborative HTML5 gene list enrichment analysis tool. BMC Bioinformatics. 2013;14:128

46. Kuleshov MV, et al. Enrichr: a comprehensive gene set enrichment analysis web server 2016 update. Nucleic Acids Res. 2016;44(W1):W90-W97.

47. Karakikes I, et al. Small molecule-mediated directed differentiation of human embryonic stem cells toward ventricular cardiomyocytes. Stem Cells Transl Med. 2014;3(1):18-31.

48. Ferruzzi J, Bersi MR, Humphrey JD. Biomechanical phenotyping of central arteries in health and disease: advantages of and methods for murine models. Ann Biomed Eng. 2013;41(7):1311-1330. 